\title{
Pyoderma gangrenosum - a guide to diagnosis and management 新
}

\author{
Authors: Christina George, ${ }^{A}$ Florence Deroide ${ }^{B}$ and Malcolm Rustin ${ }^{C}$
}

Pyoderma gangrenosum (PG) is a reactive non-infectious inflammatory dermatosis falling under the spectrum of the neutrophilic dermatoses. There are several subtypes, with 'classical PG' as the most common form in approximately $85 \%$ cases. This presents as an extremely painful erythematous lesion which rapidly progresses to a blistered or necrotic ulcer. There is often a ragged undermined edge with a violaceous/ erythematous border. The lower legs are most frequently affected although PG can present at any body site. Other subtypes include bullous, vegetative, pustular, peristomal and superficial granulomatous variants. The differential diagnosis includes all other causes of cutaneous ulceration as there are no definitive laboratory or histopathological criteria for PG. Underlying systemic conditions are found in up to $50 \%$ of cases and thus clinicians should investigate thoroughly for such conditions once a diagnosis of PG has been made. Treatment of PG remains largely anecdotal, with no national or international guidelines, and is selected according to severity and rate of progression. Despite being a well-recognised condition, there is often a failure to make an early diagnosis of PG. This diagnosis should be actively considered when assessing ulcers, as prompt treatment may avoid the complications of prolonged systemic therapy, delayed wound healing and scarring.

KEYWORDS: Dermatology, pyoderma gangrenosum, neutrophilic dermatosis, ulcer

\section{Pyoderma gangrenosum}

Pyoderma gangrenosum (PG) is a reactive non-infectious inflammatory dermatosis falling under the spectrum of the neutrophilic dermatoses, which includes Sweet's syndrome and Behcet's syndrome. The incidence is thought to be approximately 0.63 per 100,000 with the median age at presentation of 59 years. ${ }^{1}$ The sex incidence ranges from being equal, ${ }^{2}$ to females being predominantly affected in up to $76 \%$ of cases. ${ }^{3}$ There appears to be a genetic component with cases of PG clustering in families and siblings. ${ }^{4}$

Authors: A dermatology registrar, Royal Free Hospital, London, UK; ${ }^{B}$ consultant in histopathology, Royal Free Hospital, London, UK;

C professor of dermatology, Royal Free Hospital, London, UK

\section{Clinical features}

Classical PG presents most commonly as an extremely painful erythematous lesion which rapidly progresses to a blistered or necrotic ulcer. There is often a ragged undermined edge with a violaceous/erythematous border. ${ }^{1}$ The lower legs are most frequently affected although PG can present at any body site. The lesion may be precipitated by minor trauma, a phenomenon known as 'pathergy'. ${ }^{6}$ Indeed PG lesions are all too often misdiagnosed as simple non-healing ulcers and patients undergo debridement which can result in catastrophic deterioration of the condition through this pathergic response. The condition predominantly affects adults but childhood cases are rarely reported. ${ }^{7}$ Although there may be some families with PG and inherited syndromes in which PG is a feature, the majority of patients do not have a family history of the condition.

Most cases of PG are of the classic ulcerative type (approximately $85 \%$ ), but other subtypes include bullous, vegetative, pustular, peristomal and superficial granulomatous variants, with subtypes of PG sometimes transitioning from one form to another. ${ }^{9}$ The differential diagnosis has to include all other causes of cutaneous ulceration as there are no definitive laboratory or histopathological criteria for PG. PG may also involve extracutaneous sites affecting the eyes (scleritis, corneal ulceration,) lungs (aseptic pulmonary nodules) and the spleen.

\section{Classical PG}

The most common form of PG presents as a rapidly progressive painful ulcer with a violaceous undermined edge (Fig 1).

\section{Bullous PG}

This form presents with rapidly evolving painful superficial vesicles and bullae arising in waves, often coalescing together most commonly on the arms. Histologically this shares similarities with Sweet's syndrome. A haematological malignancy should be sought as these are identified in up to $70 \%$ cases. $^{10}$

\section{Pustular PG}

This form is most commonly seen in the context of flaring inflammatory bowel disease and presents with painful pustules on a background of erythema, often on the extensor surfaces. ${ }^{11}$

\section{Granulomatous superficial $P G$}

Otherwise known as vegetative PG, this subtype usually progresses more slowly and presents with verrucous and ulcerative lesions. 


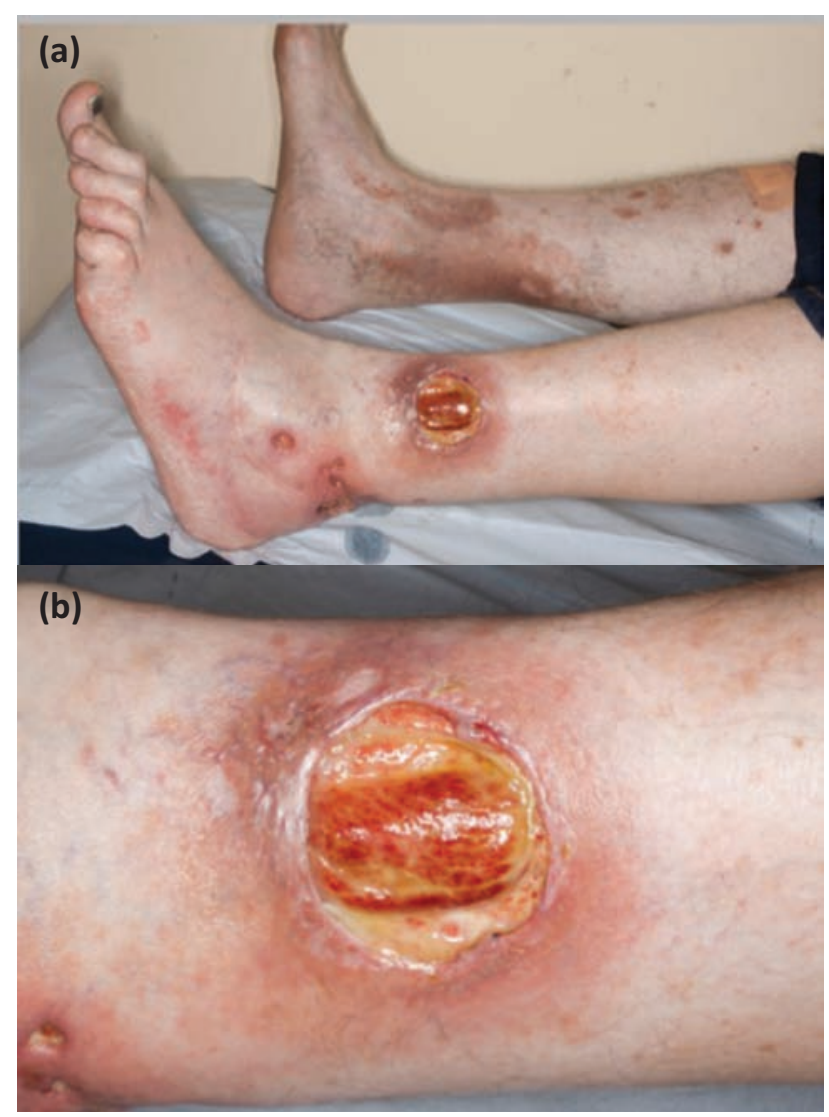

Fig 1. Rapidly progressive painful ulcer

These patients are less likely to have an underlying systemic condition and do not usually require systemic treatment. ${ }^{12}$

\section{Peristomal PG}

This variant probably results from a pathergic response to trauma from faecal irritation or secondary to appliances on the skin and is most frequently seen in the context of stomas in patients with inflammatory bowel disease. ${ }^{13}$

\section{Malignant pyoderma}

This is an important but rare clinical variant that presents with destructive ulceration typically affecting the upper torso, head and neck. Lesions do not display the violaceous edge seen in classical PG and the condition is not associated with systemic disease. ${ }^{15}$

\section{Aetiology}

Underlying systemic conditions are found in up to $50 \%$ of cases and so it is imperative to try and identify such conditions once a diagnosis of PG has been made. ${ }^{14}$ Most frequently associated conditions include inflammatory bowel disease in up to $30 \%$ of cases, rheumatoid arthritis and seronegative arthritides in up to $10 \%$ of cases, haematological malignancies or monoclonal gammopathies (in particular immunoglobulin A gammopathy) in $5 \%$ of cases and other malignancies in 5\%. Other systemic conditions have also more rarely been associated with $P G$, including those of chronic infection and inflammation. ${ }^{1,16}$ Drugs such as propylthiouracil, tyrosine kinase inhibitors, TNF $\alpha$ inhibitors and granulocyte-colony stimulating factor have been implicated but the underlying disease for which the medication has been prescribed may be the triggering factor. ${ }^{17}$

PG associated syndromes have been described and these include PG with cystic acne and hidradenitis suppurativa (PASH), ${ }^{18}$ PG with pyogenic arthritis and acne (PAPA), ${ }^{19}$ and $\mathrm{PG}$ with pyogenic arthritis, acne and hidradenitis suppurativa (PAPASH). ${ }^{20}$

\section{Pathophysiology}

The pathogenesis of PG remains unclear however it is recognised that neutrophils play a key role in the disease process. ${ }^{18}$ Upregulation of a number of key proinflammatory and neutrophil chemotactic factors within lesional skin have been identified and these include IL-1 $\beta$, IL-17, TNF $\alpha$, IL-8, IL-6, IL-17 and IL-23. IL-8 has been demonstrated to produce PG in animal models, ${ }^{22}$ it is also induced in fibroblasts of PG ulcers ${ }^{23}$ and its associated ligands are over-expressed in PG. ${ }^{24}$ There is also increased matrix metalloproteinase (MMP) expression, in particular MMP 9 and 10 which could contribute to poor healing ${ }^{25}$ together with clonal expansion of T-cells being identified in the skin and serum of patients with PG. ${ }^{26}$ However, the exact role of lymphocytes in the pathogenesis of PG has yet to be elucidated.

A genetic basis for $P G$ has so far been documented in the syndromic presentations with mutations in the PSTP1P1/CD2BP1 gene in PAPA and PASH syndromes. Normally pyrin inhibits inflammasome activation but the PSTPIP1 mutant inhibits the anti-inflammatory effect of pyrin and this results in the release of pro-inflammatory cytokines. ${ }^{27}$ The results of more sophisticated exome sequencing and next generation sequencing in other patients with PG are awaited.

\section{Histological Findings}

PG remains a clinical and sometimes challenging diagnosis and although histology of skin biopsies can be supportive, the main value of the skin biopsy is to exclude other causes of cutaneous ulceration and to allow specimens to be sent for bacterial, mycobacterial and fungal cultures. The biopsy should include the active border of the ulcer and penetrate deep to subcutaneous tissues. Patients should be warned that such a surgical procedure will invariably cause an enlargement of the ulcer, as well as potentially inducing a pathergic immunological response to trauma.

The histological findings can be variable and depend on the site of the biopsy and age of the lesion. In classic ulcerative PG there may be ulceration of the epidermis and dermis associated with an intense neutrophilic infiltrate, neutrophilic pustules and abscess formation (Fig 2). ${ }^{28}$ Different histological findings will be seen depending on the clinical variant. Vasculitis is sometimes identified histologically but this may be secondary to the ulceration. If identified, causes of true vasculitis and infection should be investigated. ${ }^{29}$

\section{Diagnosis}

Thus far there are no validated, established diagnostic clinical or pathological criteria to diagnose PG. Su et al have proposed a diagnostic tool requiring two major and two minor criteria (see Table 1), maintaining PG as a diagnosis of exclusion. ${ }^{30}$ More recently, Maverakis et al have proposed new criteria based on a consensus of international experts, requiring one major and four minor criteria (see Table 2). ${ }^{31}$ This is yet to be widely adopted, but no longer renders PG as a diagnosis of exclusion which may thus provide an improved diagnostic tool. 


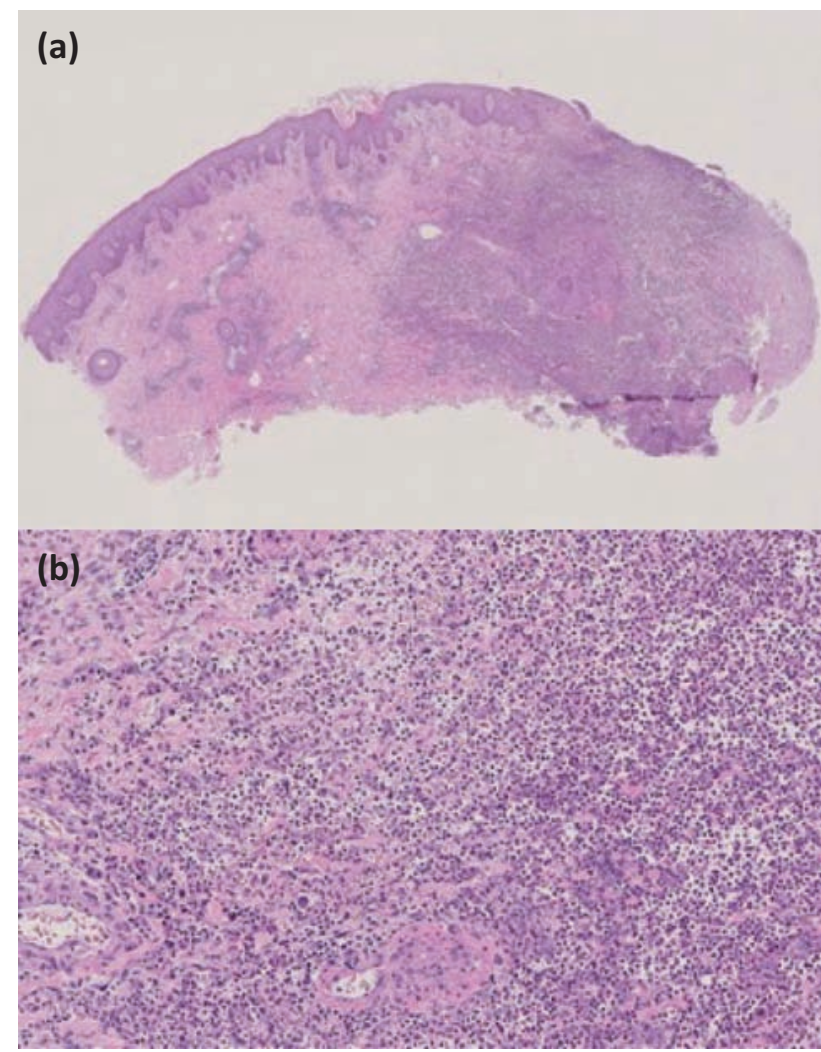

Fig 2. Histological findings with an intense neutrophilic infiltrate, neutrophilic pustules and abscess formation

\section{Differential diagnosis}

Other causes of cutaneous ulcers should be considered. These include arterial and venous disease, haematological causes (sickle cell disease, cryoglobulinaemia, anti-phospholipid syndrome), vascular occlusion, vasculitis, infections, calciphylaxis, drug-induced ulceration, primary or metastatic tumours, hypertension (Martorell ulcer) and other inflammatory disorders including cutaneous Crohn's disease.

How to approach the patient

A thorough history is key with specific enquiry regarding possible pathergic response to minor or major trauma, as well as a history

\section{Table 1. Diagnostic tool for pyoderma gangrenosum}

\section{Major criteria}

Rapid progression of a painful necrolytic cutaneous ulcer with an irregular, violaceous and undermined border

Other causes of cutaneous ulceration excluded

\section{Minor criteria}

History of pathergy or cribriform scarring clinically Associated systemic disease (inflammatory bowel disease, arthritis, IgA gammopathy or underlying malignancy)

Classic histopathological findings

Treatment response (rapid response to systemic steroid treatment - 50\% improvement in 1 month)
Table 2. Improved diagnostic tool for pyoderma

\section{gangrenosum}

\section{Major criteria}

Biopsy of ulcer edge demonstrating neutrophilic infiltrate

\section{Minor criteria}

Exclusion of infection

Pathergy

History of inflammatory bowel disease or inflammatory arthritis

History of papule, pustule or vesicle ulcerating within 4 days of appearing

Peripheral erythema, undermining border, and tenderness at ulceration site

Multiple ulcerations, at least one on anterior lower leg

Cribriform or 'wrinkled paper' scar(s) at healed ulcer sites

Decreased ulcer size within 1 month of initiating

immunosuppressive medication(s)

of pain, rapid progression, symptoms suggestive of infection or systemic disease and a detailed drug history. Clinical examination of the ulcer as well as a full body examination is essential and a skin biopsy as suggested above.

Laboratory investigations should include a full blood count, erythrocyte sediment rate, $\mathrm{C}$-reactive protein, liver and renal function tests, protein electrophoresis, urinary Bence Jones protein, a full hepatitis screen, a vasculitic screen, cryoglobulins if the history is suggestive and a coagulation screen to investigate for thrombotic causes of ulceration. A faecal calprotectin is advised if there is a clinical suspicion of inflammatory bowel disease. A chest x-ray is also indicated as a baseline screen prior to systemic therapy and computed tomography (CT) if there is a suspicion of underlying malignancy.

\section{Treatment}

Treatment of PG remains largely anecdotal with only two randomised controlled trials (RCT), and is therefore based largely

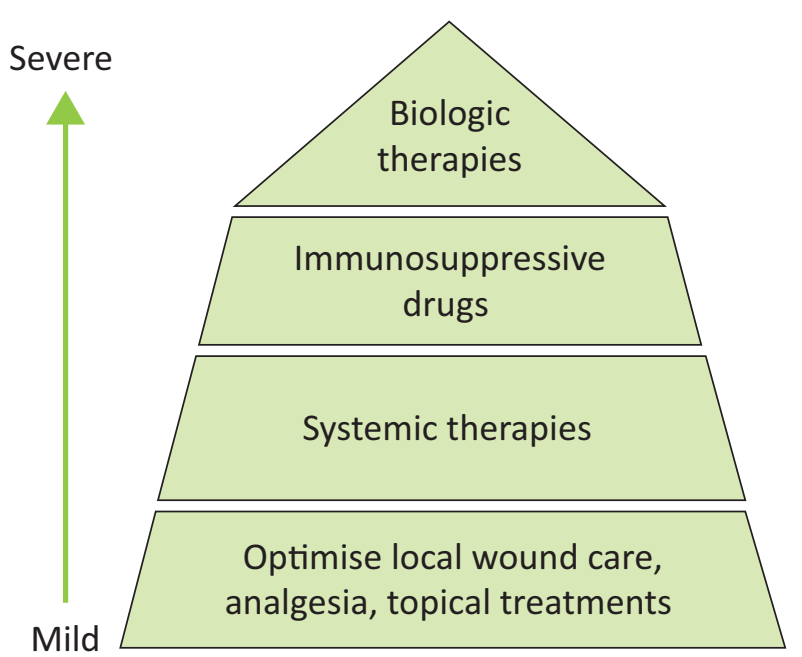

Fig 3. Treatment choice based on the severity and extent of pyoderma gangrenosum 


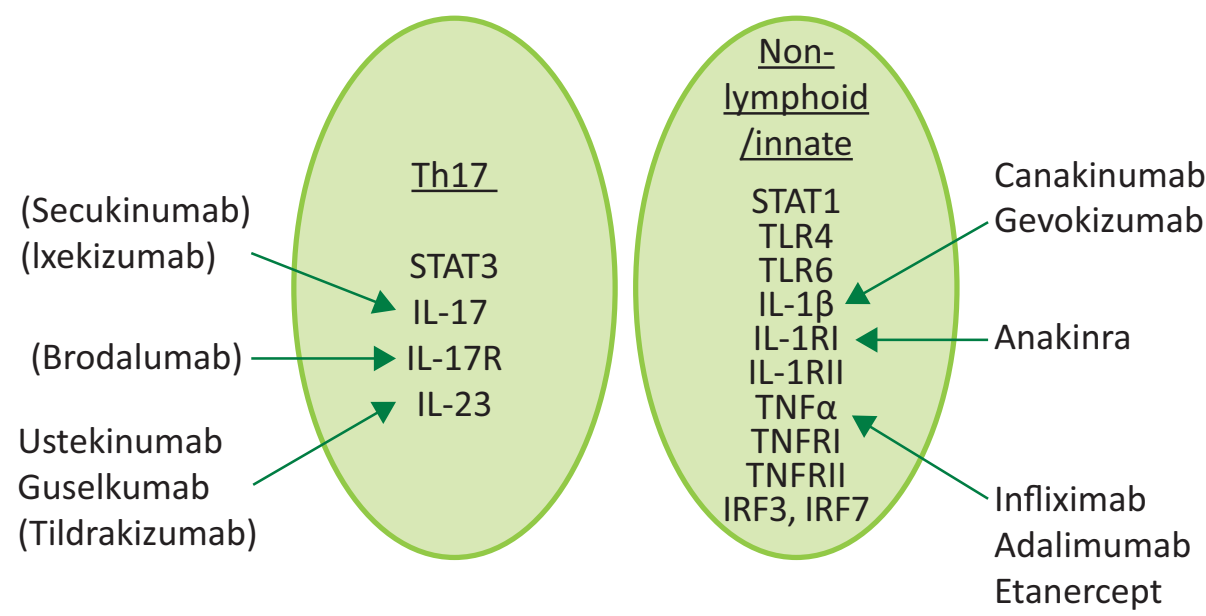

Fig 4. Biologic therapy as a treatment of pyoderma gangrenosum targeting a number of cytokines on case series and poorly evidenced publications. ${ }^{32,33}$ With no national or international guidelines, management is therefore challenging, and treatment choice is based on the severity and extent of the PG (Fig 3). ${ }^{34}$

First-line treatment is aimed at optimising local wound care, particularly important in cases of PG arising on the leg, where wound healing can be delayed by vascular disease. Supportive therapy with appropriate dressings, compression (if arterial insufficiency has been excluded) and adequate analgesia are all essential to optimise healing. Potent topical corticosteroids and tacrolimus ointment applied to the ulcer surface are useful and intralesional injections of corticosteroid into the erythematous active border may be considered. ${ }^{35}$

In more severe disease, systemic therapy is required. Oral corticosteroids $(0.5-1 \mathrm{mg} / \mathrm{kg} /$ day) are the mainstay of treatment and are used to gain rapid control. Ciclosporin can be used either alone or in combination with corticosteroids as a steroid-sparing agent, in cases where prolonged treatment is required. ${ }^{36}$ In a multicentre RCT of 121 patients, prednisolone $0.75 \mathrm{mg} / \mathrm{kg} / \mathrm{day}$ (maximum dose $75 \mathrm{mg}$ ) was compared to ciclosporin $4 \mathrm{mg} / \mathrm{kg} /$ day (maximum dose $400 \mathrm{mg}$ ) and only $50 \%$ of patients achieved remission at 6 months and there was no significant difference between the two monotherapies. ${ }^{32}$ Other systemic treatments utilised with varying success include colchicine, sulphasalazine, dapsone, minocycline, apremilast and thalidomide. Pulsed intravenous methylprednisolone can be useful in initiating a rapid response given together with immunosuppressive drugs such as methotrexate, mycophenolate mofetil, cyclophosphamide, azathioprine and high dose intravenous immunoglobulin.

There is now a growing body of evidence to support biologic therapy as a treatment of $\mathrm{PG}^{37}$ targeting a number of cytokines but there is no consensus as to whether these treatments should be administered only when other treatments have failed or as first line therapies in severe PG (Fig 4).

Thus far infliximab has the largest body of evidence to support its early use in PG. In a RCT investigating the use of infliximab (5 $\mathrm{mg} / \mathrm{kg}$ intravenous) versus placebo, $69 \%$ of patients demonstrated clinical improvement at week six after just one infusion. ${ }^{38} \mathrm{~A}$ retrospective observational study has shown faster wound healing in a significant proportion of patients treated with infliximab and adalimumab versus oral corticosteroids alone, ${ }^{39}$ further supporting the argument that biologic therapy should be considered as an early treatment strategy. Adalimumab has shown efficacy in achieving wound healing in recalcitrant $\operatorname{cases}^{40}$ and there are case reports demonstrating success with etanercept, ${ }^{41}$ ustekinumab, ${ }^{43}$ anakinra $^{43}$ and canakinumab. ${ }^{44}$

\section{Summary}

Despite being a well-recognised condition there is often a failure to make an early diagnosis of PG. It is important for all clinicians to be aware of this condition and to actively consider PG when assessing patients with ulcers, as appropriate and prompt treatment at an early stage of the disease may avoid the complications of prolonged systemic treatment, delayed wound healing and scarring.

\section{References}

1 Langan SM, Groves RW, Card TR, Gulliford MC. Incidence, mortality, and disease associations of pyoderma gangrenosum in the United Kingdom: a retrospective cohort study. J Invest Dermatol 2012;132:2166-70.

2 Ahronowitz I, Harp J, Shinkai K. Etiology and management of pyoderma gangrenosum: a comprehensive review. Am J Clin Dermatol 2012;13:191-211.

3 Binus AM, Qureshi AA, Li VW, Winterfield LS. Pyoderma gangrenosum: a retrospective review of patient characteristics, comorbidities and therapy in 103 patients. Br J Dermatol 2011;165:1244-50.

4 Alberts JH, Sams HH, Miller JL et al. Familial ulcerative pyoderma gangrenosum: a report of 2 kindred. Cutis 2002;69:427-30.

5 Schwaegerle SM, Bergfeld WF, Senitzer D et al. Pyoderma gangrenosum: A review. J Am Acad Dermatol 1988;18:559-68.

6 Sassolas B, Le Ru Y, Plantin P et al. Pyoderma gangrenosum with pathergic phenomenon in pregnancy. $\mathrm{Br}$ ] Dermatol 2000;142:827-8.

7 Graham JA, Hansen KK, Rabinowitz LG et al. Pyoderma gangrenosum in infants and children. Pediatr Dermatol 1994;11:10-7.

8 Sakiyama M, Kobayashi T, Nagata Y et al. Bullous pyoderma gangrenosum: A case report and review of the published work. J Dermatol 2012:39:1010-5.

9 Weenig RH, Davis MD, Dahl PR et al. Skin ulcers misdiagnosed as pyoderma gangrenosum. N Engl J Med 2002;347:1412-8.

10 Perry HO, Winkelmann RK. Bullous pyoderma gangrenosum and leukemia. Arch Dermatol 1972;106:901-5.

11 Shankar S, Sterling JC, Rytina E. Pustular pyoderma gangrenosum. Clin Exp Dermatol 2003;28:600-3. 
12 Wilson-Jones E, Winkelmann RK. Superficial granulomatous pyoderma: a localized vegetative form of pyoderma gangrenosum. J Am Acad Dermatol 1988;18:511-21.

13 Wu XR, Mukewar S, Kiran RP et al. Risk factors for peristomal pyoderma gangrenosum complicating inflammatory bowel disease. J Crohns Colitis 2013;7:e171-7.

14 Bennett ML, Jackson JM, Jorizzo JL et al. Pyoderma gangrenosum. A comparison of typical and atypical forms with an emphasis on time to remission. Case review of 86 patients from two institutions. Medicine (Baltimore) 2000;79:37-46.

15 Erdi H, Anadolu R, Pişkin G et al. Malignant pyoderma: A clinical variant of pyoderma gangrenosum. Int J Dermatol 1996;35:8113.

16 Callen JP, Jackson JM. Pyoderma gangrenosum: an update. Rheum Dis Clin North Am 2007;33:787-802.

17 Wang JY, French LE, Shear NH, Amiri A, Alavi A. Drug-induced pyoderma gangrenosum: a review. Am J Clin Dermatol 2017; 19:67-77.

18 Braun-Falco M, Kovnerystyy O, Lohse P, Ruzicka T. Pyoderma gangrenosum, acne, and suppurative hidradenitis (PASH) - a new autoinflammatory syndrome distinct from PAPA syndrome. J Am Acad Dermatol 2012;66:409-15.

19 Lindor NM, Arsenault TM, Solomon H, Seidman CE, McEvoy MT. A new autosomal dominant disorder of pyogenic sterile arthritis, pyoderma gangrenosum, and acne: PAPA syndrome. Mayo Clinic Proc 1997;72:611-5.

20 Marzano AV, Trevisan V, Gattorno M et al. Pyogenic arthritis, pyoderma gangrenosum, acne, and hidradenitis suppurativa (PAPASH): a new autoinflammatory syndrome associated with a novel mutation of the PSTPIP1 gene. JAMA Dermatol 2013;149:762-4.

21 Magro CM, Kiani B, Li J et al. Clonality in the setting of Sweet's syndrome and pyoderma gangrenosum is not limited to underlying myeloproliferative disease. J Cutan Pathol 2007;34:526-34.

22 Oka M, Berking C, Nesbit M et al. Interleukin-8 overexpression is present in pyoderma gangrenosum ulcers and leads to ulcer formation in human skin xenografts. Lab Invest 2000;80:595-604.

23 Oka M. Pyoderma gangrenosum and interleukin 8. Br J Dermatol 2007:157:1279-81.

24 Marzano AV, Fanoni D, Antiga E et al. Expression of cytokines, chemokines and other effector molecules in two prototypic autoinflammatory skin diseases, pyoderma gangrenosum and Sweet's syndrome. Clin Exp Immunol 2014;178:48-56.

25 Bister V, Makitalo L, Jeskanen L et al. Expression of MMP-9, MMP-10 and TNF-alpha and lack of epithelial MMP-1 and MMP-26 characterize pyoderma gangrenosum. J Cutan Pathol 2007:34:889-98.

26 Brooklyn TN, Williams AM, Dunnill MG et al. T-cell receptor repertoire in pyoderma gangrenosum: evidence for clonal expansions and trafficking. $\mathrm{Br}$ ] Dermatol 2007;157:960-6.

27 Tallon B, Corkill M. Peculiarities of PAPA syndrome. Rheumatology (Oxford) 2006;45:1140-3.

28 Weedon D. Weedon's Skin pathology, 3rd ed. Elsevier, 2010:195244.

29 Weenig RH, Davis MD, Dahl PR et al. Skin ulcers misdiagnosed as pyoderma gangrenosum. N Engl J Med 2002;347:1412-8.
30 Su WP, Davis MD, Weenig RH, Powell FC, Perry HO. Pyoderma gangrenosum: clinicopathologic correlation and proposed diagnostic criteria. Int J Dermatol 2004;43:790-800.

31 Maverakis E, Ma C, Shinkai K et al. Diagnostic criteria of ulcerative pyoderma gangrenosum. A Delphi consensus of international experts. JAMA Dermatol 2018;154:461-6.

32 Ormerod AD, Thomas KS, Craig FE et al. Comparison of the two most commonly used treatments for pyoderma gangrenosum: results of the STOP GAP randomised controlled trial. BM] 2015;350:h2958.

33 Brooklyn TN, Dunnill MG, Shetty A et al. Infliximab for the treatment of pyoderma gangrenosum: a randomised, double blind, placebo controlled trial. Gut 2006;55:505-9.

34 Miller J, Yentzer BA, Clark A, Jorizzo JL, Feldman SR. Pyoderma gangrenosum: a review and update on new therapies. J Am Acad Dermatol 2010;62:646-54.

35 Wenzel J, Gerdsen R, Phillipp-Dormston W, Bieber T, Uerlich M. Topical treatment of pyoderma gangraenosum. Dermatology 2002:205:221-3.

36 Reichrath J, Bens G, Bonowitz A, Tilgen W. Treatment recommendations for pyoderma gangrenosum: an evidence-based review of the literature based on more than 350 patients. J Am Acad Dermatol 2005;53:273-83

37 Agarwal A, Andrews JM. Systematic review: IBD-associated pyoderma gangrenosum in the biologic era, the response to therapy. Aliment Pharmacol Ther 2013;38:563-72.

38 Brooklyn TN, Dunnill MG, Shetty A et al. Infliximab for the treatment of pyoderma gangrenosum: a randomised, double blind, placebo-controlled trial. Gut 2006;55:505-9.

39 Arguelles-Arias F, Castro-Laria L, Lobaton T et al. Characteristics and treatment of pyoderma gangrenosum in inflammatory bowel disease. Dig Dis Sci 2013;58:2949-54.

40 Fonder MA, Cummins DL, Ehst BD, Anhalt GJ, Meyerle JH. Adalimumab therapy for recalcitrant pyoderma gangrenosum. J Burns Wounds 2006;5:e8.

41 Charles CA, Leon A, Banta MR, Kirsner RS. Etanercept for the treatment of refractory pyoderma gangrenosum: a brief series. Int J Dermatol 2007:46:1095-9.

42 Fahmy M, Ramamoorthy S, Hata T, Sandborn WJ. Ustekinumab for peristomal pyoderma gangrenosum. Am J Gastroenterol 2012;107:794-5.

43 Brenner M, Ruzicka T, Plewig G, Thomas P, Herzer P. Targeted treatment of pyoderma gangrenosum in PAPA (pyogenic arthritis, pyoderma gangrenosum and acne) syndrome with the recombinant human interleukin-1 receptor antagonist anakinra. Br J Dermatol 2009;161:1199-201.

44 Geusau A, Mothes-Luksch N, Nahavandi H et al. Identification of a homozygous PSTPIP1 mutation in a patient with a PAPA-like syndrome responding to canakinumab treatment. JAMA Dermatol 2013;149:209-15

Address for correspondence: Dr Christina George, Royal Free Hospital, Pond St, Hampstead, London NW3 2QG, UK. Email: cgeorge2@nhs.net 